\title{
Personal Safety is More Important Than Cost of Damage During Robot Failure*
}

\author{
Obehioye Adubor \\ Carnegie Mellon University \\ Pittsburgh, PA 15213 \\ USA \\ oadubor@gmail.com
}

\author{
Rhomni St. John \\ U. of Massachusetts Amherst \\ Amherst, MA, 01003 \\ USA \\ rcstjohn@umass.edu
}

\author{
Aaron Steinfeld \\ Carnegie Mellon University \\ Pittsburgh, PA 15213 \\ USA \\ steinfeld@cmu.edu
}

\begin{abstract}
As robots become more common in everyday life it will be increasingly important to understand how non-experts will view robot failure. In this study, we found that severity of failure seems to be tightly coupled with perceived risk to self rather than risk to the robot's task and object. We initially thought perceived severity would be tied to the cost of damage. Instead, participants placed falling drinking glasses above a laptop when rating the severity of the failure. Related results reinforce the primacy of personal safety over the financial cost of damage and suggest the results were tied to proximity to breaking glass.
\end{abstract}

\section{KEYWORDS}

Human-robot interaction; failure; safety

\section{INTRODUCTION}

The main goal of this study was to assess possible factors contributing to a non-expert's reaction to robot failure. We were particularly interested in how bystanders would react to failure events and whether they would be willing to intervene. Past work in human trust in robots suggests financial risk is important (e.g., [1]), but more insights are needed on whether other types of risk are important during robot autonomy failure.

Of immediate interest are risk factors that might suppress or alter intervention by bystanders. Concerns about personal risk in close quarters have been seen for autonomous vehicles [2].

\section{METHODS}

This Amazon Mechanical Turk study ( $n=261)$ explored how four failure scenario factors influenced intervention. There were 36 video clips showing permutations of these four factors. Specifically, we manipulated whether a human was present (not present, off-screen operator, human working with the robot), damage (food, drinking glasses, laptop), robot behavior (wobbling

Permission to make digital or hard copies of part or all of this work for personal or classroom use is granted without fee provided that copies are not made or distributed for profit or commercial advantage and that copies bear this notice and the full citation on the first page. Copyrights for third-party components of this work must be honored. For all other uses, contact the Owner/Author(s). Copyright is held by the owner/author(s).

HRI'17, March 6-9, 2017, Vienna, Austria.

ACM ISBN 978-1-4503-4336-7/17/03.

DOI: http://dx.doi.org/10.1145/3029798.3036649 arm motion, no wobble), and prior task success (failure on first instance, success on first and failure on second).

Participants were shown a single, randomly assigned video clip showing a Baxter robot failing to place an object into a receptacle and items falling towards the floor. The view was framed so impact could not be seen and there was no audio. Participants then responded to questions on severity of the failure, willingness to intervene, and intervention type.

\section{RESULTS}

Detailed analyses are still underway, but several initial findings are immediately apparent. First and foremost, participants placed the drinking glasses above the laptop when rating the severity of the failure $(F(2,254)=4.75, p<0.01)$, with no difference between the laptop and the food.

A related result was seen for three safety questions framed around the participant's hypothetical distance from the robot (20', 10 ', and 3'), which showed a large drop for only the drinking glass at 3'. Likewise, participants showed greater willingness to press an E-stop button when the robot was handling the drinking glasses, as compared to the other items $(F(2,258)=4.14, p<0.05)$.

\section{CONCLUSIONS}

Based on these results, it is reasonable to assume that perceived personal risk takes priority over perceived damage when considering intervention. This is, at a smaller scale, comparable to aversion to personal risk when near crimes, natural disasters, and other more extreme events. This needs to be explored with in-person studies with more realistic perceived risk.

\section{ACKNOWLEDGMENTS}

This work was funded by a grant (IIS-1552256) from the National Science Foundation.

\section{REFERENCES}

[1] Munjal Desai, Mikhail Medvedev, Marynel Vázquez, Sean McSheehy, Sofia Gadea-Omelchenko, Christian Bruggeman, Aaron Steinfeld, and Holly Yanco. 2012. Effects of changing reliability on trust of robot systems. In Proceedings of ACM/IEEE International Conference on Human-Robot Interaction (HRI '12). ACM, New York, NY. DOI=http://dx.doi.org/10.1145/2157689.2157702

[2] Dirk Rothenbücher, Jamy Li, David Sirkin, Brian Mok, and Wendy Ju. 2015. Ghost driver: a platform for investigating interactions between pedestrians and driverless vehicles. In Adjunct Proceedings of the International Conference on Automotive User Interfaces and Interactive Vehicular Application. ACM, New York, NY. DOI=http://dx.doi.org/10.1145/2809730.2809755 